\title{
Rare Unruptured Thin-Cap Fibroatheroma within Left Main Coronary Artery in Acute Coronary Syndrome
}

\author{
Hemanth Harish Ponnana ${ }^{1}$ Lalita Nemani ${ }^{1}$ \\ ${ }^{1}$ Department of Cardiology, Nizam's Institute of Medical Sciences, \\ Hyderabad, India
}

Ind J Car Dis Wom 2019;4:204-206

\begin{abstract}
Address for correspondence Dr. Hemanth Harish, Department of Cardiology, Nizam's Institute of Medical Sciences, Hyderabad 500082, India (e-mail: hemu2030@gmail.com).
\end{abstract}

\author{
Abstract \\ Keywords \\ - unruptured thin-cap \\ fibroatheroma \\ - LMCA \\ - OCT \\ - acute coronary \\ syndrome
}

\begin{abstract}
Optical coherence tomography-guided percutaneous coronary angioplasties (PCI) for acute coronary syndrome is useful not only to the good stent opposition but also to the underlying pathophysiology and morphology of the plaque. Here we are reporting a case of anterior myocardial infarction thin-cap fibroatheroma (TCFA) in left main coronary artery with mid left anterior descending artery tight fibrofatty lesion. The cap of the atheromatous lesion was unruptured. This phenomenon of unruptured thincap fibroatheroma in the left main coronary artery in acute coronary syndrome has seldom been reported previously.
\end{abstract}

\section{Introduction}

Cardiac imaging is gaining importance in percutaneous coronary angioplasties $(\mathrm{PCI})$ both in patients with acute coronary syndromes and in complex chronic stable angina. Intravascular ultrasound (IVUS) and optical coherent tomography (OCT) are used to know the underlying plaque morphology before stenting, to know the complexity of the intervention as well as the chances of distal embolization. They are useful even after stenting to know the approximation and complications related to stenting. For vulnerable plaque detection, OCT scores over IVUS..$^{1-3}$

Thin-cap fibroatheroma (TCFA), which is one of the features of plaque vulnerability, is usually seen at the culprit occlusive or tighter lesion site. Here, we are reporting TCFA in the left main coronary artery (LMCA), other site than the culprit lesion site.

\section{Case Report}

Our patient is a 65-year-old male patient presented with complaints of sudden-onset of chest pain, which was radiating to left arm, associated with shortness of breath functional class 3 on August 3, 2019, following which he was taken to a hospital where he was diagnosed as a case of evolved anterior wall myocardial infarction. He underwent coronary angiogram subsequently and reported as ectatic coronaries and was kept on medical management for the same. One month later, he had similar symptoms and reported to our center; on evaluation at our center his electrocardiogram showed normal sinus rhythm with qs complexes in V1 to V5 precordial leads and 2D echocardiogram showed regional wall motion abnormality in left anterior descending artery (LAD) territory with moderate left ventricle (LV) dysfunction (ejection fraction [EF] 40\%). However, his troponin T was normal. Hence, he was diagnosed as post-myocardial infarction (MI) angina. Previous coronary angiography (CAG) film review showed improper opacification of coronaries due to nonselective injections. We repeated coronary angiogram on September 5, 2019, which showed mid-LAD tight stenosis and left circumflex artery (LCX) diseases. He was subsequently taken up for percutaneous intervention under OCT guidance. OCT images were acquired using a nonocclusive technique with the illumin optis mobile system (dragonfly catheter and optis mobile system, near-infrared light imaging). The artery was cleared of blood by continuously flushing with iodixanol 320 (Visipaque, GE Health Care, Cork, Ireland) at a flow rate of $3.0 \mathrm{~mL} / \mathrm{s}$. Mid-LAD showed significant tight lesion on conventional coronary angiogram ( - Fig. 1). Subsequently, OCT run showed an interesting finding in LMCA (-Fig. 2). A thin-cap fibroatheromatous (TCFA) plaque seen in LMCA (-Figs. 2 and 3) without significant obstruction.

Usually, TCF represents a vulnerable plaque. The point discussed, in this case, was where the obstructive mid-LAD lesion or the TCF of LMCA is the culprit lesion. As the post-MI angina is due to the obstructive mid-LAD lesion, we did stenting to published online December 26, 2019
DOI https://doi.org/ $10.1055 / \mathrm{s}-0039-3402823$
(C)2019 Women in Cardiology and Related Sciences
License terms

() (1) $\ominus \circledast$ 


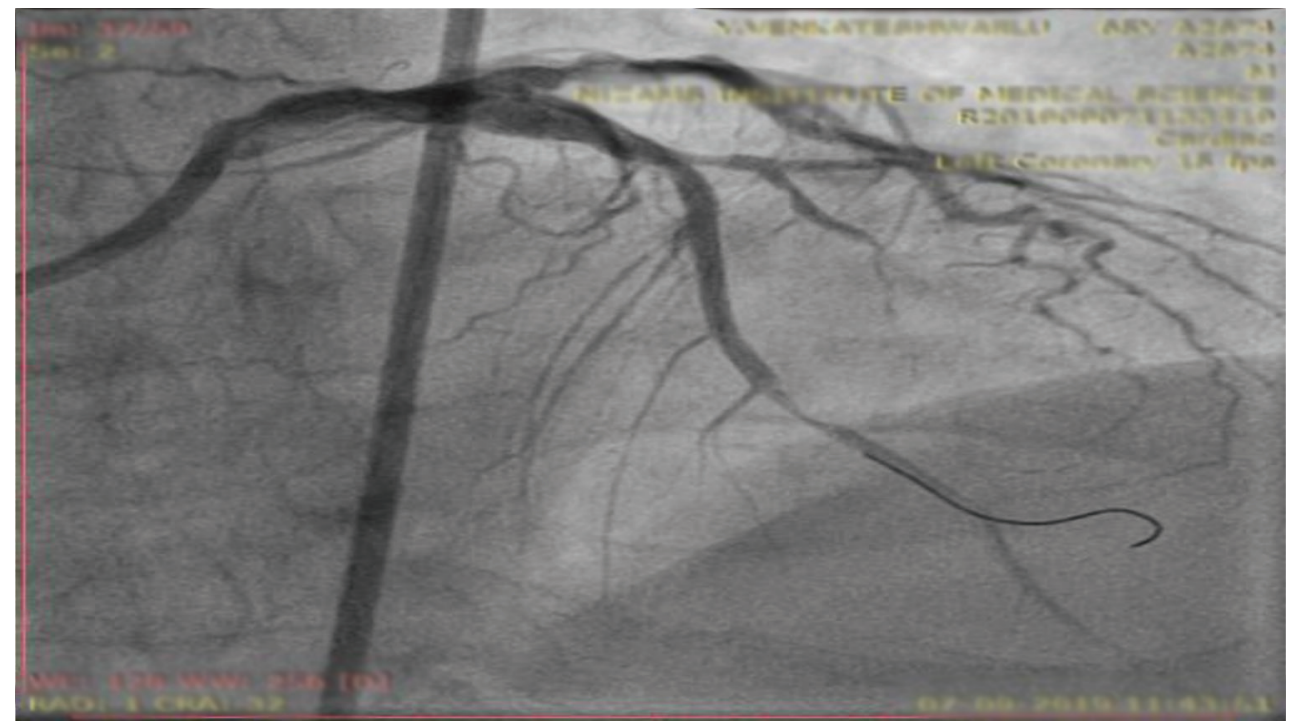

Fig. 1 Conventional angiogram image showing significant mid-LAD stenosis.

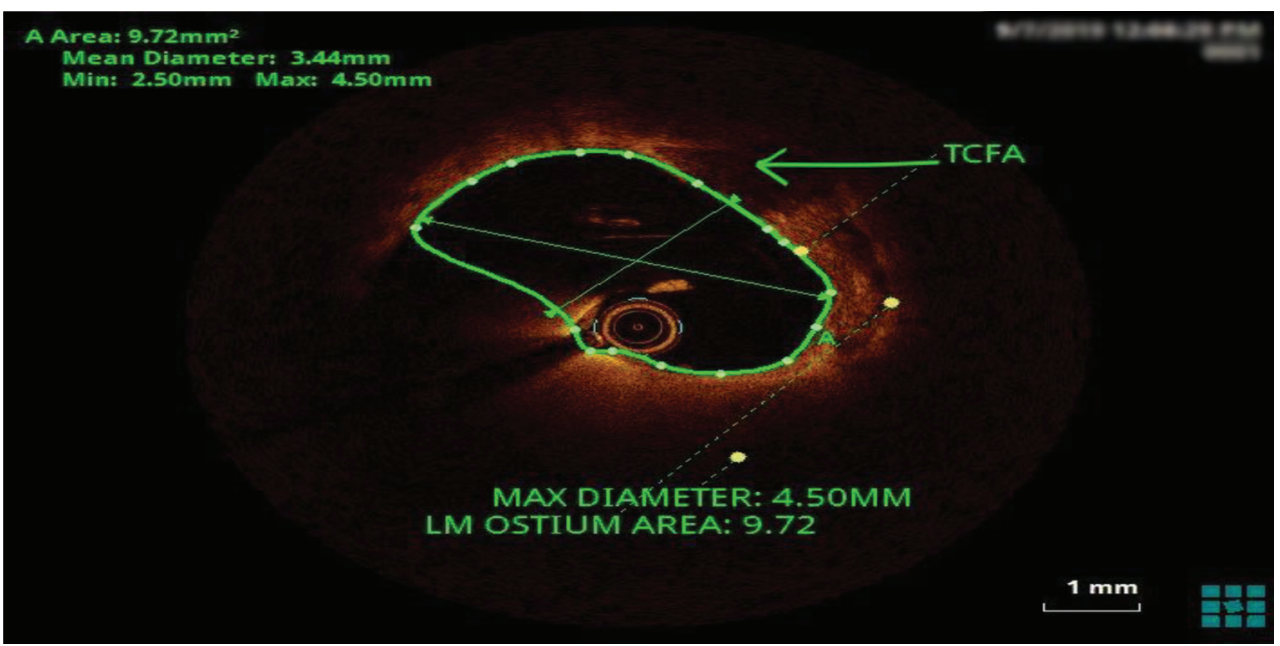

Fig. 2 Optical coherence tomography of left main coronary artery.

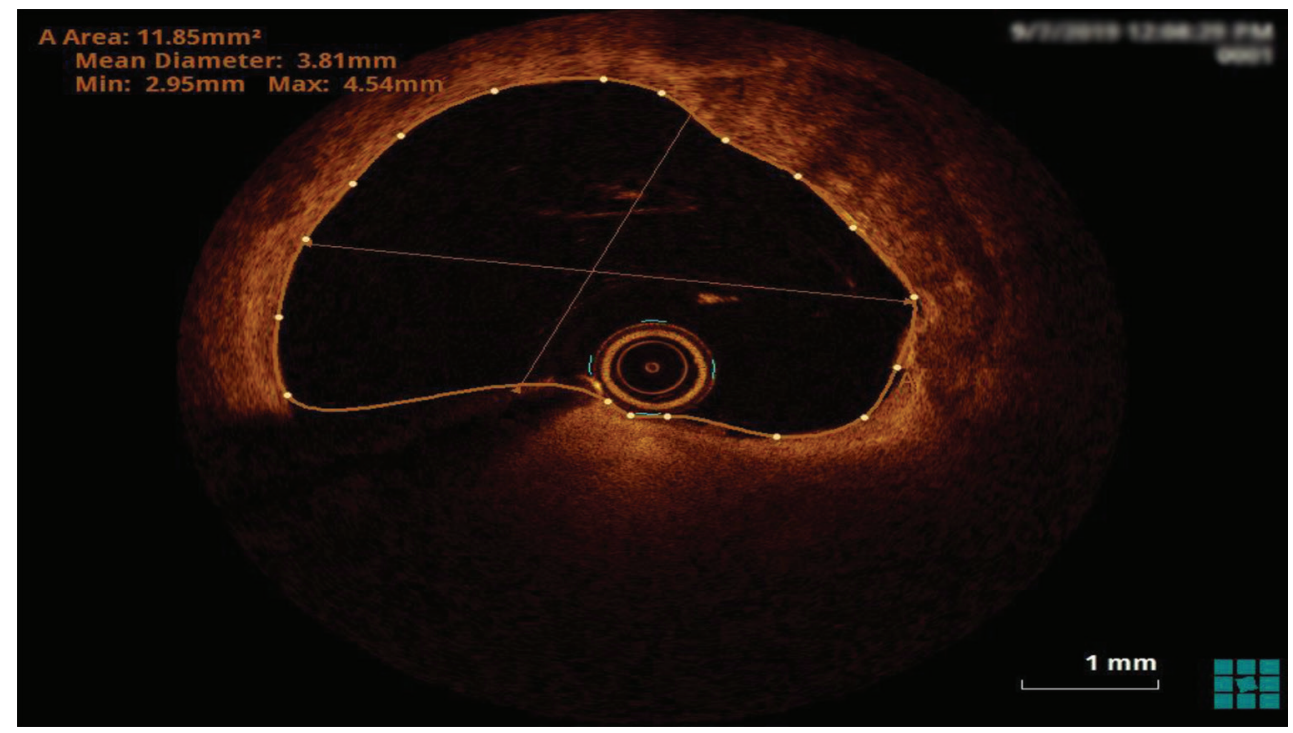

Fig. 3 Zoomed view of the optical coherence tomography image of left main coronary artery depicting thin-cap fibroatheroma. 


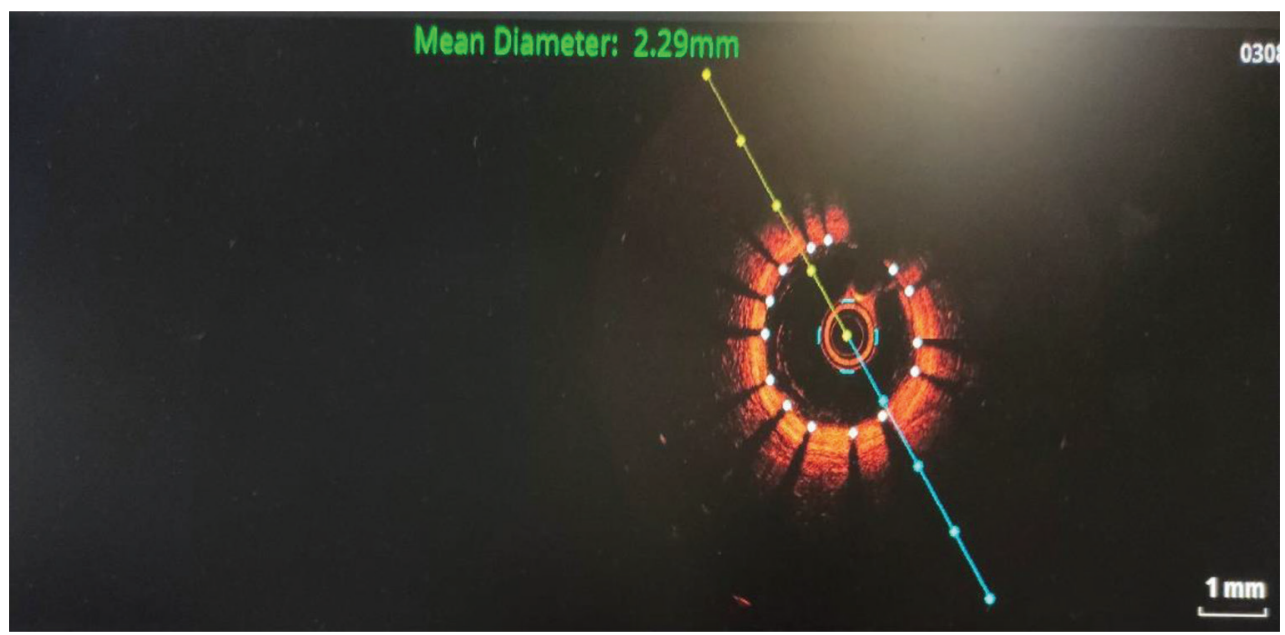

Fig. 4 Post-percutaneous coronary angioplasty mid-LAD.

mid-LAD with $2.5 \times 28 \mathrm{~mm}$ drug eluting stent (DES) (-Fig. 4) and poststenting OCT run was taken. Uniform stent opposition was confirmed and also showed that TCF in LMCA was not disrupted. He was continued on DAPT (aspirin and prasugrel) with a high dose of rosuvastatin along with ACEI and $\beta$-blockers. He was discharged three days after the procedure and is in close follow-up since then.

\section{Discussion}

A conventional coronary angiogram is a $2 \mathrm{D}$ imaging modality and is bound to miss numerous nonobstructive plaques and fibroatheromas. Although IVUS and coronary angioscopy also help in identifying and delineating fibrous cap atheromas (TCF), OCT is more superior to detect them. ${ }^{4,5}$ As OCT provides better spatial resolution with good delineation of 4-20 $\mu \mathrm{m}$ size, the histological components of coronary plaques and measurement for TCF are very reliable.,6

TCFA was defined as fibrous cap $<65 \mu \mathrm{m}$ overlying a necrotic core. TCFA represents the vulnerability of the plaque. ${ }^{7}$ According to Fabio Tavora, in autopsy series, they demonstrated ruptured TCFA at the site of thrombotic occlusion of the culprit lesion in only $25 \%$, and a ruptured plaque was present along with culprit lesion in $38 \%$ and in $44 \%$ of cases in the nonculprit artery. ${ }^{8}$ Tanimoto et al also showed that multiple small ruptured plaques were demonstrated in the distal sites of the culprit lesion containing artery. ${ }^{9}$ In the present case, there was an unruptured vulnerable plaque in the LMCA, proximal to the culprit lesion of mid-LAD.

The vulnerability of the plaque not only depends on TCFA but also on the necrotic lipid-rich core, abundant infiltrating macrophages and neovascularization. ${ }^{10,11}$ Our case demonstrated only TCFA without underlying necrotic core and neovascularization.

\section{Conclusion}

We are reporting this case as thin-cap fibroatheromas is rare in LMCA, that too unruptured plague, when compared with proximal segments of LAD, LCX, and RCA in acute coronary syndrome.

\section{Conflict of Interest}

None.

\section{References}

1 Carrizo S, Salinas P, Jimenez-Valero S, Moreno R. Utility of optical coherence tomography to assess a hazy intracoronary image after percutaneous coronary intervention. Korean Circ J 2013;43(1):44-47

2 Regar E, Ligthart J, Bruining N, van Soest G. The diagnostic value of intracoronary optical coherence tomography. Herz 2011;36(5):417-429

3 Bouma BE, Tearney GJ, Yabushita H, et al. Evaluation of intracoronary stenting by intravascular optical coherence tomography. Heart 2003;89(3):317-320

4 Bourantas CV, Garg S, Naka KK, Thury A, Hoye A, Michalis LK. Focus on the research utility of intravascular ultrasoundcomparison with other invasive modalities. Cardiovasc Ultrasound 2011;9(1):2

5 Kubo T, Imanishi T, Takarada S, et al. Assessment of culprit lesion morphology in acute myocardial infarction: ability of optical coherence tomography compared with intravascular ultrasound and coronary angioscopy. J Am Coll Cardiol 2007;50(10):933-939

6 Stamper D, Weissman NJ, Brezinski M. Plaque characterization with optical coherence tomography. J Am Coll Cardiol 2006;47(8, Suppl):C69-C79

7 Virmani R, Burke AP, Kolodgie FD, Farb A. Pathology of the thin-cap fibroatheroma: a type of vulnerable plaque. J Interv Cardiol 2003;16(3):267-272

8 Tavora F, Cresswell N, Li L, Fowler D, Burke A. Frequency of acute plaque ruptures and thin cap atheromas at sites of maximal stenosis [in Portuguese]. Arq Bras Cardiol 2010;94(2):143-149

9 Tanimoto T, Imanishi T, Tanaka A, et al. Various types of plaque disruption in culprit coronary artery visualized by optical coherence tomography in a patient with unstable angina. Circ J 2009;73(1):187-189

10 Papaioannou TG, Kalantzis C, Katsianos E, Sanoudou D, Vavuranakis M, Tousoulis D. Personalized assessment of the coronary atherosclerotic arteries by intravascular ultrasound imaging: hunting the vulnerable plaque. J Pers Med 2019;9(1):8

11 Tarkin JM, Dweck MR, Evans NR, et al. Imaging atherosclerosis. Circ Res 2016;118(4):750-769 